\title{
Accuracy of a rapid diagnostic test on the diagnosis of malaria infection and of malaria - attributable fever during low and high transmission season in Burkina Faso
}

\author{
Zeno Bisoffi*1,2, Sodiomon B Sirima ${ }^{3}$, Joris Menten ${ }^{4}$, Cristian Pattaro5, Andrea Angheben'1, Federico Gobbi1,2, \\ Halidou Tinto6, Claudia Lodesani7, Bouma Neya², Maria Gobbo ${ }^{1}$ and Jef Van den Ende ${ }^{8}$
}

\begin{abstract}
Background: Malaria management policies currently recommend that the treatment should only be administered after laboratory confirmation. Where microscopy is not available, rapid diagnostic tests (RDTs) are the usual alternative. Conclusive evidence is still lacking on the safety of a test-based strategy for children. Moreover, no formal attempt has been made to estimate RDTs accuracy on malaria-attributable fever. This study aims at estimating the accuracy of a RDT for the diagnosis of both malaria infection and malaria - attributable fever, in a region of Burkina Faso with a typically seasonal malaria transmission pattern.
\end{abstract}

Methods: Cross-sectional study. Subjects: all patients aged > 6 months consulting during the study periods. Gold standard for the diagnosis of malaria infection was microscopy. Gold standard for malaria-attributable fever was the number of fevers attributable to malaria, estimated by comparing parasite densities of febrile versus non-febrile subjects. Exclusion criteria: severe clinical condition needing urgent care.

Results: In the dry season, 186/852 patients with fever (22\%) and 213/1,382 patients without fever (15\%) had a Plasmodium falciparum infection. In the rainy season, this proportion was 841/1,317 (64\%) and 623/1,669 (37\%), respectively. The attributable fraction of fever to malaria was $11 \%$ and $69 \%$, respectively. The RDT was positive in $113 /$ 400 (28.3\%) fever cases in the dry season, and in $443 / 650$ (68.2\%) in the rainy season. In the dry season, the RDT sensitivity and specificity for malaria infection were $86 \%$ and $90 \%$ respectively. In the rainy season they were $94 \%$ and $78 \%$ respectively. In the dry season, the RDT sensitivity and specificity for malaria-attributable fever were $94 \%$ and $75 \%$, the positive predictive value (PPV) was $9 \%$ and the negative predictive value (NPV) was $99.8 \%$. In the rainy season the test sensitivity for malaria-attributable fever was $97 \%$ and specificity was $55 \%$. The PPV ranged from $38 \%$ for adults to $82 \%$ for infants, while the NPV ranged from $84 \%$ for infants to over $99 \%$ for adults.

Conclusions: In the dry season the RDT has a low positive predictive value, but a very high negative predictive value for malaria-attributable fever. In the rainy season the negative test safely excludes malaria in adults but not in children.

\section{Background}

The adoption of artemisinin-based combination therapy (ACT) for malaria in most endemic countries, and the availability of new diagnostic tools, such as rapid diagnostic tests (RDTs), have led the World Health Organization (WHO) to recommend a modified approach to malaria

\footnotetext{
* Correspondence: zeno.bisoffi@sacrocuore.it

1 Centre for Tropical Diseases, S. Cuore Hospital, 37024 Negrar (Verona), Italy Full list of author information is available at the end of the article
}

management. The previous policy indicated the presumptive treatment for malaria of all patients with fever, unless another obvious cause was found. The increased cost of new treatments, as well as the concern for the potential selection of drug resistant Plasmodium falciparum strains, prompted a more selective approach. The new policy recommends that malaria treatment should only be administered after laboratory confirmation. This recommendation had initially been limited to older chil- 
dren and adults, but the new edition of WHO guidelines, released on March 9 th, 2010 [1], recommends to extend the test-based policy to children under five, too, as was strongly suggested by some authors [2]. There is no universal agreement on this new policy, though, and other authors argue that moving to a generalized test-based policy may not be safe, due to the sub-optimal performance of RDTs under field conditions, involving the risk of missing true malaria cases, with potentially fatal consequences in young children [3]. Moreover, recent field studies showed that the adherence of health workers to the test result was poor, with many patients being treated for malaria even after a negative test result, causing an obvious waste of resources [4-6].

Despite many published papers on RDT performance in different countries [7-20], important evidence gaps remain to be filled: a) the safety of a test-based strategy, especially for children, has not yet been fully demonstrated; b) evidence is needed on effective strategies to ensure an adequate compliance of prescribers with the test result; c) the utility of RDTs in areas of great seasonal variation in malaria transmission has not yet been adequately addressed, and the optimal policy might not be the same throughout the year [21]; d) although the accuracy of RDTs for malaria infection has been studied, no attempt has been made so far to estimate how accurately RDTs predict malaria - attributable fever.

A recent randomized trial on RDT-based versus presumptive treatment [4] aiming at assessing RDT safety on the same study population was partly frustrated, precisely, by a particularly poor adherence to the negative test result.

This paper addresses the third and fourth knowledge gaps, aiming at assessing RDT accuracy in an area of Burkina Faso with great seasonal variation in transmission intensity and at estimating, in both seasons, how RDTs perform as predictors of malaria-attributable fever. The two issues are clearly linked, as the proportion of fevers attributable to malaria may vary greatly according to the season.

RDTs are designed to detect malaria infection. In an endemic area many individuals are asymptomatic carriers of malaria parasites. A patient with fever and with malaria parasites in blood may be a case of malaria, or an individual with another cause of fever, and with incidental parasitaemia. No malaria test, including RDTs, is designed to discriminate between the two conditions, and indeed, any febrile patient with malaria parasites in blood, detected with whatever method, should be treated for malaria. However, if the parasite density is high, the fever is more likely to be due to malaria. Traditional microscopy provides a quantitative estimate of the parasite density, while RDTs do not, barely indicating the presence or absence of malaria parasites. A false negative
RDT result may be of no consequence, if it fails to detect an incidental, low parasitaemia in a patient with another cause of fever. Paradoxically, a positive result might be harmful in the same patient, by confirming a clinical suspicion of malaria. RDTs based on HRP-2 protein have shown a variable accuracy for malaria infection in field studies, with microscopy taken as the gold standard. A recent review reported results ranging from $87.5 \%$ to $100 \%$ for sensitivity and from $52 \% \%$ to $99.5 \%$ for specificity [15], the latter being also hampered by the tendency of the test to remain positive even weeks after a successfully treated malaria $[10,22]$. No study so far has formally attempted to assess RDTs as predictors of malaria-attributable fever.

\section{Research questions}

This study primarily aimed at estimating how accurately RDTs predict malaria-attributable fever in the low and high transmission seasons. The main steps are outlined below:

1. The prevalence of malaria infection was assessed among febrile and non-febrile patients presenting at primary health care centres at the end of the dry and of the rainy season.

2. The fraction of fever episodes attributable to malaria infection (attributable fraction, AF) was determined in both seasons.

3. The accuracy of a rapid diagnostic test on malaria infection was evaluated in both seasons.

4. The performance of the RDT as a predictor of malaria-attributable fever was estimated in both seasons.

\section{Methods}

A cross-sectional study was carried out in May and in October, 2006, in 10 primary care health centres of the provinces of Bobo Dioulasso and Banfora, south-west of Burkina Faso, an area with stable malaria and with a seasonal transmission pattern. The lowest transmission takes place at the end of the dry season (April-May), and the highest transmission at the end of the rainy season (October). The study sites were selected with convenience criteria, as described elsewhere [4].

All (febrile and non-febrile) patients $>6$ months consulting one of the study sites for any clinical problem during the study periods (24 $4^{\text {th }}$ April to $19^{\text {th }}$ May and $2^{\text {nd }}$ to $20^{\text {th }}$ October, 2006) and giving their (or their guardians') written informed consent were consecutively submitted to a standardized medical examination, and to thick and thin film, by specifically trained research assistants. The research assistants were trained by the study investigators and by professional laboratory staff from an Italian referral centre (see below) for three days preceding each study period: training included the correct execution of malaria 
smears (thick and thin film) and the execution and reading of the RDT.

Exclusion criteria were: severe clinical condition needing urgent care. An axillary temperature was obtained upon recruitment for all patients using an electronic digital thermometer (accuracy $\pm 0.1^{\circ} \mathrm{C}$, certified CE 0197). Fever was defined as an axillary temperature $\geq 37.5^{\circ} \mathrm{C}$.

A random sample of the febrile patients, in both seasons, was also submitted to a malaria RDT (Paracheck Device), in the context of the randomized trial cited above [4]. The febrile patients submitted to the RDT were, therefore, the same patients as in the trial, about half of the total febrile patients included in the study. The necessary sample size of the study was related to the primary outcome of the randomized trial: at least 2,000 febrile patients, plus all non-febrile patients presenting during the same periods.

The reference test was malaria microscopy executed by highly experienced staff from the Centre for Tropical Diseases (CTD) of S. Cuore Hospital of Negrar, Verona, a reference centre in Italy. The thick and thin films were coded locally and transported daily to a central laboratory (Centre Muraz, Bobo Dioulasso) for Giemsa staining by local staff, supervised by two senior microscopists from the CTD. Reading was done by the senior microscopists who were masked to the result of the RDT as well as to the clinical status (febrile or non-febrile) of the patients. A number of microscopic fields corresponding to $200 \mathrm{WBC}$ were read in the thick film. The parasite density was calculated (for P. falciparum only) in the conventional way according with WHO criteria. A double blind cross reading of a random sample of 300 slides (thick plus thin films) was carried out in order to check for interobserver variability, as a double reading of all the $>5,000$ slides was not feasible.

The RDT used in the study was the test Paracheck Device (Orchid Biomedical Systems, Goa, India, batches 21,226 and 31,333, expiry dates June 2007 and January 2008 , respectively), which detects the $P$. falciparum specific HRP-2 protein. The tests were individually sealed, were transported and stored according to the manufacturer's instructions, and were opened a few minutes before use. They were performed and read by the trained research assistants, coded and stored for future control. For positive results, the time of appearance of the positive band was recorded. RDT reading was checked every evening by the senior microscopists.

\section{Statistical analysis}

Data were double-entered at Centre Muraz, Bobo Dioulasso, with Epi Info software (EpiInfo, CDC Atlanta, version 3.3.2). Data analyses were carried out with $R$ 2.8.0 (R Development Core Team. R: A Language and Environment for Statistical Computing. R Foundation for Statisti- cal Computing. Vienna, Austria, 2008. ISBN: 3-90005107-0), and Stata 10.1 (StataCorp LP, College Station, TX 77845 USA) statistical packages.

The primary aim of the analysis was to estimate how RDTs predict malaria-attributable fever in the high and low transmission seasons. The main conceptual steps are summarized below, and detailed in the following paragraphs.

1. The prevalence of (falciparum) malaria infection was assessed in febrile and non-febrile patients.

2. The proportion of fevers attributable to malaria was estimated, stratifying by parasite density class and age group. The attributable fraction (AF) was defined as the proportion of fevers, among infected patients, that would not have occurred in the absence of malaria infection. Formulas used for AF calculation are reported below.

3. The diagnostic accuracy of RDTs for malaria infection by parasite density and age group was calculated.

4. The attributable fractions and the calculated diagnostic accuracy of RDT for malaria infection were combined to obtain an estimate of the sensitivity and specificity of RDT for malaria-attributable fever, accounting for both the expected increasing sensitivity of RDT for infection and the higher likelihood of a fever to be due to malaria at higher parasite densities.

All analyses were performed on the rainy and dry season data, separately.

The prevalence of falciparum malaria was estimated as the proportion of patients with a positive slide for $P$. falciparum asexual forms (any parasite density) among febrile and non-febrile patients. The AF of fever to malaria infection was estimated from the odds-ratios obtained from logistic regression modelling according to methods described for case-control studies [23] where patients with fever (axillary temperature $\geq 37.5^{\circ} \mathrm{C}$ ) were defined as cases and patients without fever and without recent (3 days) fever history as controls. The odds-ratio (OR) of fever in each stratum of parasite density $(0,1-400,401-$ $4000,4001-40,000$ and $>40,000$ parasites $/ \mu \mathrm{l}$, the upper limit of each stratum roughly corresponding to $1 / 10,000$ parasites/RBC, 1/1,000, 1/100 and >1/100) and agegroup (6-11 months, $1-4$ years, $5-14$ years, $\geq 15$ years) were calculated. The AF was then estimated from the ORs, as $A F=(O R-1) / O R$, for each cross-classification of parasite density and age (20 strata) for the rainy season. The number of fever cases and of positive malaria films was too low to determine the AF by age groups for the dry season. Consequently, for this season AFs were estimated by parasite density class only, adjusting for age, from the adjusted odds-ratios (aOR) as $\mathrm{AF}=(\mathrm{aOR}-1) /$ aOR. In addition to the AF, the population attributable fraction (PAF) was also estimated, defined as the proportion of fevers attributable to malaria infection among all patients with fever, to assess the burden of disease in the 
whole population, and obtained by multiplying the AF by the prevalence of malaria infection among all febrile patients (PrevMal): $\mathrm{PAF}=\mathrm{AF}($ PrevMal).

RDT sensitivity, specificity, positive and negative predictive value (PPV, NPV) were estimated for malaria infection on the subset of febrile patients undergoing the RDT, and with microscopy results taken as the gold standard. As for the AF, sensitivity and specificity were calculated for each cross-classification of parasite density and age (20 strata) for the rainy season and for each parasite density stratum for the dry season. In addition, PPV and NPV of the RDT were assessed in febrile patients during the rainy and dry seasons. Confidence intervals were estimated with the Wilson's score method[24]. To assess how the agreement between RDT and microscopy was influenced by variables other than parasite density (such as season, age and sex), a logistic regression model was used where the outcome was a dichotomous variable taking values of 1 in case of method agreement or 0 in case of disagreement.

Based on RDT performances on malaria infection at each level of parasite density and age, its accuracy was subsequently assessed on malaria-attributable fever.

The AF-based approach does not allow classifying each individual febrile case with a positive slide as having clinical malaria, or simple malaria infection with another cause of fever. However, through this approach it is possible to estimate the number of malaria-attributable fevers at each stratum of parasite density and age, by multiplying the number of febrile cases in each stratum by the respective AF.

The number of true positive RDT results was then calculated in each stratum as the product of the number of malaria-attributable fevers and the probability of a RDT positive test result for febrile patients in the stratum. The total number of RDT true positives was the sum of the RDT true positives in all strata, as in formulas reported in Table a1a. In a similar way, the number of false positives (Table b1b), false negatives (Table c1c), and true negatives (Table d1d) were estimated. The RDT sensitivity was then calculated as the ratio of true positives (Table a1a) to the sum of true positives and false negatives (Table $a+b 1 a+$ b), and the specificity as the ratio of true negatives (Table d1d) to the sum of true negatives and false positives $($ Table $c+d 1 c+d)$. Similarly, the PPV was estimated as the number of true positives (Table ala) divided by the number of RDT positives (Table $\mathrm{a}+\mathrm{cla}+\mathrm{c}$ ), and the NPV as the number of true negatives (Table d1d) over the number of RDT negatives (Table $b+d 1 b+d)$.

As a sensitivity analysis, the assessment of RDT diagnostic accuracy was repeated using logistic regression models for the risk of fever (to calculate AF) and for the probability of testing positive at RDT for each individual patient, including malaria infection status (yes/no), log- parasite density, and linear and quadratic terms for age. For the rainy season analyses, age/infection status and age/parasite density interaction terms were also included. The product of the AF and probability for an RDT positive result was added up for all febrile patients to obtain the diagnostic accuracy of RDT for malaria-attributable fever, similar to the stratified analysis.

The study protocol was approved by the "Comite National d'Ethique" (National Ethical Committee) of Burkina Faso (N. 2006-011 of $7^{\text {th }}$ April 2006). Written informed consent was obtained through the use of an information sheet with detailed explanation of the purpose of the study and the procedures involved. Once the clinical officer had decided that a patient was eligible for inclusion, a research assistant gave the explanation in local language, in the presence of at least one independent witness. In case of agreement, the informed consent form was signed both by the patient (or one of the parents in case of minors) and by the witness. For illiterate people the signature was replaced by the fingerprint.

\section{Results}

A total of 5,759 patients eligible for inclusion (2,557 and 3,202 in the dry and rainy season, respectively) were asked their informed consent; 5,236 consenting patients were enrolled in the study (2,235 in the dry season and 3,001 in the rainy season) (Figure 1 and 2). Sixteen records $(0.3 \%)$ were then excluded from the analysis because of missing data. The non-febrile patients were further classified according to fever history in the past three days: 474 and 731 patients with fever history and 908 and 938 without fever history in the dry and rainy season, respectively (Table 2).

\section{Microscopy and RDT reading}

The inter-observer variability of microscopy reading was assessed on a randomly taken sample of 300 slides that were blindly re-read by the study microscopists. In one case, a slide previously read negative was subsequently read positive (presence of $P$. falciparum with parasite density $<50 / \mu \mathrm{L}$, confirmed by a third reading), and conversely, in another case, a previously diagnosed very low P. falciparum parasitemia $(<50 / \mu \mathrm{L})$ was missed by the second reading (the third reading confirmed the presence of $P$. falciparum asexual forms). Inter-observer variability in the assessment of parasite density was within acceptable limits: in particular, in three instances only was the class of parasite density (see below) different among observers, and the difference was of one class only. RDT reading by the research assistants presented no problem and was invariably confirmed by the study supervisors on site and by the subsequent control (every evening) by the senior microscopists. Only for seven samples (four in the dry season and three in the rainy season) the result was 
Table 1: Formulas used for the estimation of the RDT sensitivity and specificity on malaria - attributable fever

\begin{tabular}{|c|c|c|}
\hline & Malaria - attributable fevers & Fevers not attributable to malaria \\
\hline \multirow[t]{2}{*}{ RDT+ } & a) True Positives & c) False Positives \\
\hline & $\sum_{i=1}^{20} n$ FebrilePatients $_{i} \times A F_{i} \times(\% R D T+)_{i}$ & $\sum_{i=1}^{20} n_{\text {FebrilePatients }} \times\left(1-A F_{i}\right) \times(\% R D T+)_{i}$ \\
\hline \multirow[t]{3}{*}{ RDT- } & b) False Negatives & d) True Negatives \\
\hline & $\sum_{i=1}^{20} n$ FebrilePatients ${ }_{i} \times A F_{i} \times(\% R D T-)_{i}$ & $\sum_{i=1}^{20} n_{\text {FebrilePatients }} \times\left(1-A F_{i}\right) \times(\% R D T-)_{i}$ \\
\hline & $S E=\frac{a}{a+b}$ & $S P=\frac{d}{c+d}$ \\
\hline
\end{tabular}

All summations of the 20 combinations from the cross-classification of parasite density ( 5 strata: $0,1-400,401-4000,4001-40,000$ and > 40,000) and age (4 strata: 6-11 months, 1-4 years, 5-14 years, $\geq 15$ years).

reported as indeterminate, and in one case (rainy season) it was missing. All eight were then excluded from the analysis of RDT accuracy.

Prevalence of malaria infection and of positive RDT results In the dry season, $186 / 852$ patients with fever (22\%), and 213/1,382 patients without fever (15\%) had a $P$. falciparum malaria infection. Among the latter group, the proportion was $148 / 908$ (16\%) in the group reporting no

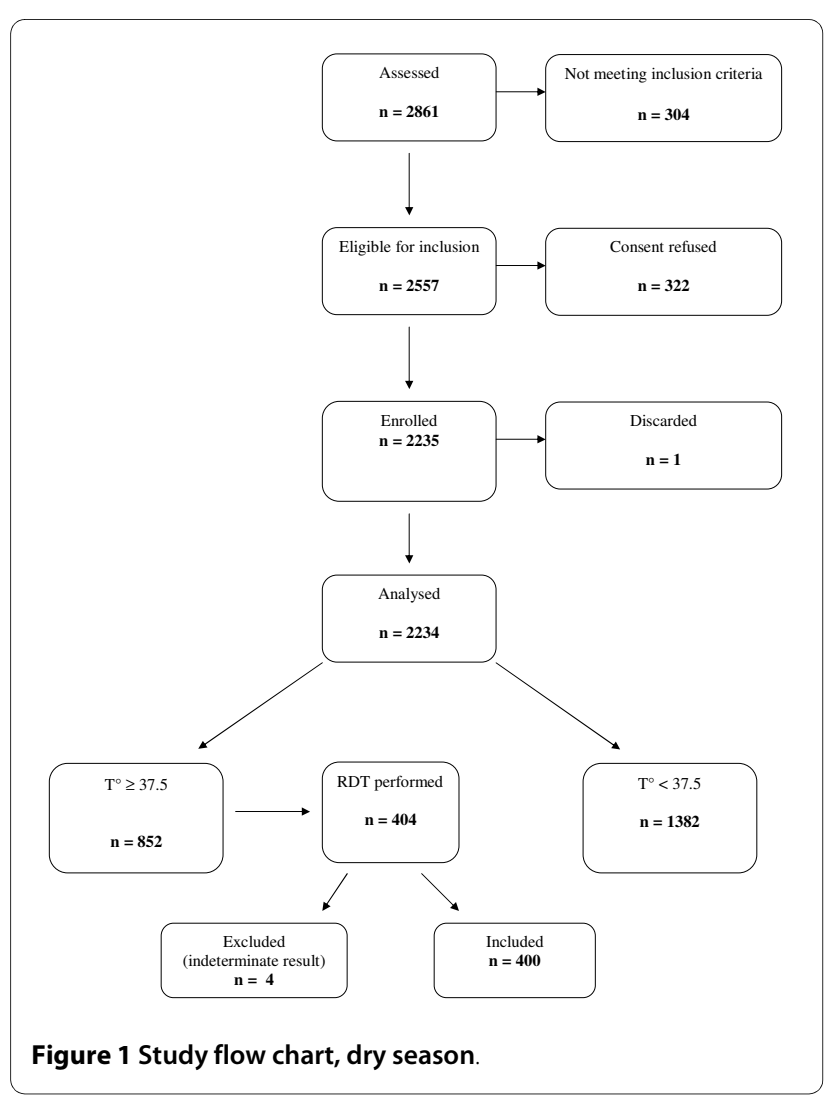

fever history in the last three days, and 65/474 (14\%) in the group reporting such history. In the rainy season, the proportion of patients with $P$. falciparum malaria infection was 841/1,317 (64\%) and 623/1,669 (37\%) in patients with and without fever, respectively; and in particular: 318/938 (34\%) in the group with no fever history and 305/ 731 (42\%) in the group with such history. The group with fever history was excluded from the calculation of the AF, in order to avoid misclassification of febrile/non-febrile

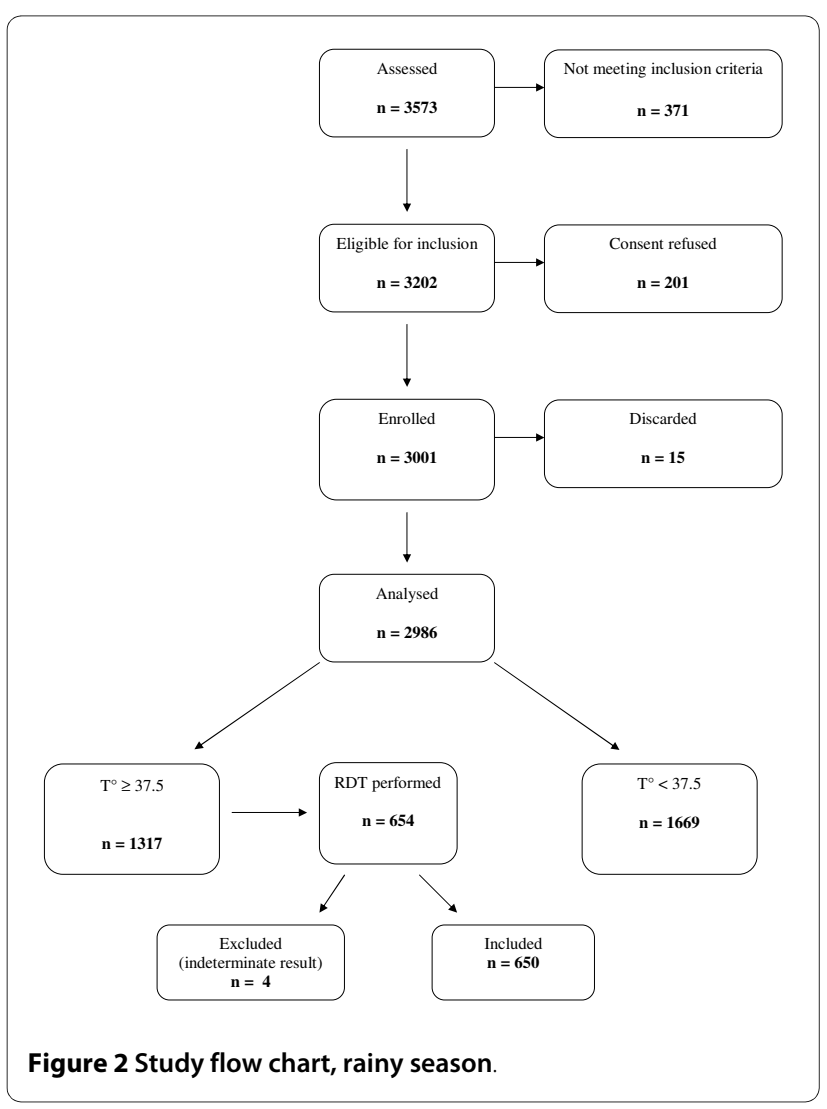


Table 2: Characteristics of febrile and non-febrile patients included in the study

\begin{tabular}{llll}
\hline & Fever & History of fever & No fever \\
\hline Dry season: $\mathrm{N}(\%)$ & & & 908 \\
- Age: mean (SD) & 852 & 474 & $22.7(18.4)$ \\
- P. falciparum infected: $\mathrm{n}(\%)$ & $11.5(14.1)$ & $22.2(18.6)$ & $148(16.3)$ \\
- median (IQR) parasite density ${ }^{\dagger}$ & $186(21.8)$ & $65(13.7)$ & $240(80-1300)$ \\
- RDT positive: $\mathrm{n} / \mathrm{N}$ tested (\%) & $360(120-2520)$ & $360(120-2760)$ & $\mathrm{NA}$ \\
Rainy season: $\mathrm{N}$ & $113 / 400^{¥}(28.3)$ & $\mathrm{NA}$ & 938 \\
- Age (mean) & 1317 & 731 & $21.2(16.8)$ \\
- P. falciparum infected: $\mathrm{n}(\%)$ & $10.7(13.4)$ & $19.2(17.4)$ & $318(33.9)$ \\
- median (IQR) parasite density ${ }^{\dagger}$ & $841(63.9)$ & $305(41.7)$ & $600(80-3320)$ \\
- RDT positive: $\mathrm{n} / \mathrm{N}$ tests (\%) & $5200(1124-42400)$ & $2720(360-15730)$ & $\mathrm{NA}$ \\
\hline
\end{tabular}

${ }^{\dagger}$ For those with any parasites.

₹ For those with axillary temperature $<37.5^{\circ} \mathrm{C}$.

¥ Seven indeterminate results (four in the dry season and three in the rainy season) and one missing result (rainy season) were excluded from the denominator of $\mathrm{N}$ tested and from subsequent calculations of RDT accuracy

patients. The RDT was positive in $113 / 400$ febrile patients in the dry season (28\%) and in $443 / 650$ febrile patients in the rainy season $(68 \%)$.

\section{Attributable fraction}

The overall population attributable fraction (PAF) of fever to malaria among patients attending health clinics (obtained by multiplying the AF with the prevalence of malaria infection) was $2 \%$ in the dry season and $44 \%$ in the rainy season. Among those infected, the AF was $11 \%$ and 69\%, respectively (Table 3 and 4).

In the dry season (Table 3), only in patients with over 4,000 parasites/ $\mu \mathrm{l}$ was a considerable proportion of fever cases attributable to malaria (AF $=24 \%$ for parasite density between 4,001 and 40,000, and $86 \%$ for parasite density $>40,000)$. Results for the rainy season are reported in Table 4 . AF was $29 \%$ for the lowest stratum of parasite density, $62 \%$ for density 401 to $4,000,76 \%$ for density 4,000 to 40,000 , and $94 \%$ for densities of $>40,000$ parasites/ $\mu$ l. A visual breakdown of the whole patient population in the two seasons is reported in Figure 3a.

When results of the rainy season were stratified by age group, it was noted that AF decreased as age increased. For children younger than five years of age a substantial proportion of fever cases was attributable to malaria even at the lowest parasite density. In infants $<1$ year old, in particular, even at the lowest parasite density $(1-400 / \mu \mathrm{l})$, the AF was very high (94\%), and consistently so at all levels of parasite density. Contrarily to all other age groups, there was no substantial difference in AF across strata of parasite density (Table 4).

A visual breakdown of under 5 versus $\geq 5$ years patient populations in the rainy season is reported in Figure $3 \mathrm{~b}$.

\section{Accuracy of RDT for malaria infection}

The RDT was positive in $113 / 400$ (28.3\%) fever cases in the dry season, and in $443 / 650(68.2 \%)$ in the rainy season (Table 2). Seven indeterminate results (four in the dry season and three in the rainy season) and one missing result (rainy season) were excluded from the denominator for calculations of RDT accuracy. Results from the logistic regression model showed, as expected, that the probability of agreement between RDT and microscopy was mostly influenced by parasite density. Season and age had little effect, while sex had no influence.

The sensitivity and specificity results of RDT for malaria infection are presented stratified by season, parasite density and age group. In the dry season (Table 5), overall sensitivity and specificity (when microscopy is taken as the gold standard) were $86 \%$ (95\% CI: 78-92\%) and $90 \%$ (95\% CI: 86-92) respectively. In the rainy season, overall sensitivity was 94\% (95\% CI: 92-96) and specificity was 78\% (95\% CI: 72-83\%) (Table 6). In both seasons, for parasite density below $400 / \mu$ l test sensitivity was $76 \%$, for densities between 400 and $400096 \%$ and $94 \%$ in the dry and rainy season respectively, and $>99 \%$ for higher densities. In one case, in the rainy season, a high parasite density $(>150,000 / \mu \mathrm{l})$ was missed by the RDT. The patient, a six-year-old boy, was diagnosed as a case of malaria (the only symptoms were high fever and vomiting), but after the RDT result he was not given any antimalarial, but an antibiotic.

The positive predictive value (PPV) for malaria infection in patients with fever was $72 \%$ (95\% CI: 63-79) in the dry season and $88 \%$ (95\% CI: 85-91) in the rainy season; the negative predictive value (NPV) was 95\% (95\% CI: 92- 
Table 3: Attributable fractions (AF) and population attributable fractions (PAF) of fever to malaria by parasite density (dry season)

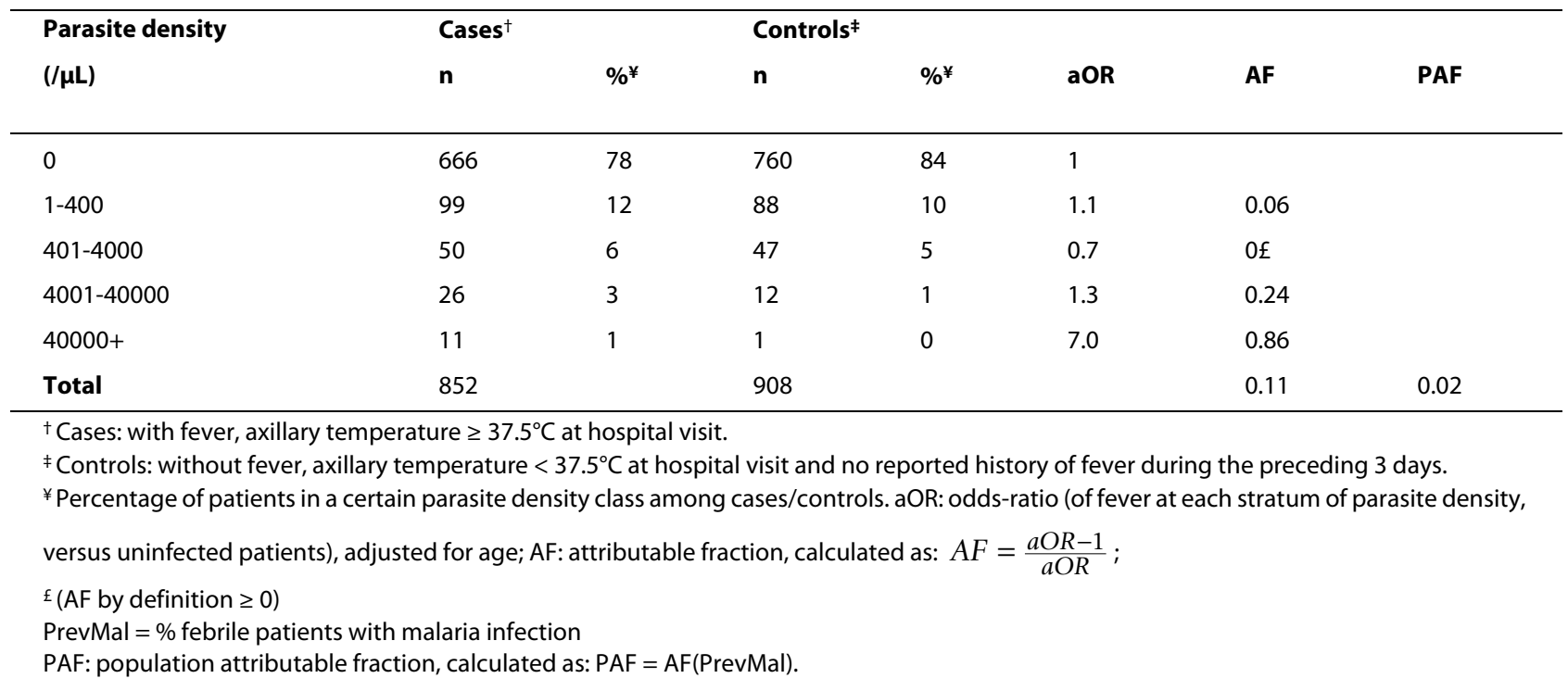

97) and $88 \%$ (95\% CI: 83-92) in the dry and rainy season, respectively.

\section{Accuracy of RDT for malaria - attributable fever}

In the dry season, RDT sensitivity and specificity for malaria-attributable fever were $94 \%$ and $75 \%$, PPV was $9 \%$, and NPV was $99.8 \%$. In the rainy season, the test sensitivity for malaria attributable fever was $97 \%$ and was consistent across age groups, while specificity ranged between 36\% (in children aged 5-14 yrs) and 78\% (in adults), and was 55\% overall. The PPV ranged from $38 \%$ in adults to $82 \%$ in infants, while the NPV ranged from $84 \%$ in infants to $>99 \%$ in adults. The overall PPV and NPV in the rainy season were $63 \%$ and $96 \%$, respectively (Table 7). The breakdown by age group and parasite density is reported in Additional files 1 and 2. Results were confirmed by the sensitivity analysis with adjusted logistic regression modelling, as shown in Additional files 3 and 4 .

\section{Discussion}

According to microscopy, in the rainy season versus the dry season, the prevalence of malaria infection in patients presenting at primary health care centres was three times higher in febrile patients and twice in non-febrile patients. In the dry season, only a small proportion of fevers were attributable to malaria. This may be surprising, but is coherent with a low transmission level, typical of the dry season. In a previous study in Burkina Faso capital city Ouagadougou during the "cold" dry season, the AF of fever to malaria infection was also exceedingly low [25]. Conversely, in the rainy season, it was observed that almost half of all fevers were attributable to malaria and this proportion was highest in infants and lowest in adults. In infants (6-11 months) in the rainy season, our results clearly show that even at the lowest parasite density the attributable fraction is close to $100 \%$ : a fever associated with the presence of malaria parasites in blood is virtually always attributable to malaria in this age group, regardless the parasite density. A similar result was found by McGuinnes et al in Ghana [26]. In children 1 to 4 years old, only about half of the cases were attributable to malaria for densities < 400, while this proportion increased with parasite density. In older children and in adults, a fever was never attributable to malaria at parasite densities $<400$. These findings are clearly relevant to the policy of RDT use to be adopted locally.

\section{RDT accuracy for malaria infection}

The test sensitivity, specificity, positive and negative predictive values (PPV, NPV) for malaria infection, as reported in Tables 5 and 6 , are within the range found by previous studies $[15,20,27]$.

Although the different test sensitivity in the dry and in the rainy season may be surprising, this difference appears to be caused almost entirely by the different mean parasite density in the two seasons: if the analysis is stratified for parasite density, the sensitivity is very similar in both seasons. The overall sensitivity was lower than $95 \%$, the minimal level recommended by the WHO. However, most false negative results occurred at the lowest parasite density. Over 400 parasites/ $\mu$ l the sensitivity was higher than $95 \%$ and approached $100 \%$ over 4,000 parasites $/ \mu \mathrm{l}$. Leaving without treatment patients with false 
Table 4: Attributable fractions (AF) and population attributable fractions (PAF) of fever to malaria by parasite density and age group (rainy season)

\begin{tabular}{|c|c|c|c|c|c|c|c|c|}
\hline \multirow{2}{*}{$\begin{array}{l}\text { Age } \\
\text { (years) }\end{array}$} & \multirow{2}{*}{$\begin{array}{l}\text { Parasite density } \\
(/ \mu \mathrm{L})\end{array}$} & \multirow{2}{*}{$\begin{array}{c}\text { Cases }^{\dagger} \\
\text { n }\end{array}$} & \multicolumn{3}{|c|}{ Controls ${ }^{\ddagger}$} & \multirow[b]{2}{*}{ OR } & \multirow[b]{2}{*}{ AF } & \multirow[b]{2}{*}{ PAF } \\
\hline & & & $\% \neq$ & $\mathbf{n}$ & $\% \neq$ & & & \\
\hline \multirow[t]{6}{*}{$<1$} & 0 & 27 & 17 & 32 & 64 & 1 & & \\
\hline & $1-400$ & 14 & 9 & 1 & 2 & 16.6 & 0.94 & \\
\hline & $401-4000$ & 40 & 25 & 9 & 18 & 5.3 & 0.81 & \\
\hline & $4001-40000$ & 41 & 26 & 4 & 8 & 12.1 & 0.92 & \\
\hline & $40000+$ & 37 & 23 & 4 & 8 & 11.0 & 0.91 & \\
\hline & Total & 159 & & 50 & & & 0.88 & 0.73 \\
\hline \multirow[t]{6}{*}{$1-4$} & 0 & 111 & 22 & 88 & 55 & 1 & & \\
\hline & $1-400$ & 40 & 8 & 14 & 9 & 2.3 & 0.56 & \\
\hline & $401-4000$ & 132 & 26 & 32 & 20 & 3.3 & 0.69 & \\
\hline & $4001-40000$ & 100 & 20 & 21 & 13 & 3.8 & 0.74 & \\
\hline & $40000+$ & 121 & 24 & 6 & 4 & 16.0 & 0.94 & \\
\hline & Total & 504 & & 161 & & & 0.77 & 0.60 \\
\hline \multirow[t]{6}{*}{$5-14$} & 0 & 77 & 28 & 66 & 48 & 1 & & \\
\hline & $1-400$ & 34 & 12 & 31 & 23 & 0.9 & $0^{f}$ & \\
\hline & $401-4000$ & 57 & 21 & 26 & 19 & 1.9 & 0.47 & \\
\hline & $4001-40000$ & 52 & 19 & 12 & 9 & 3.7 & 0.73 & \\
\hline & $40000+$ & 55 & 20 & 2 & 1 & 23.6 & 0.96 & \\
\hline & Total & 275 & & 137 & & & 0.59 & 0.43 \\
\hline \multirow[t]{6}{*}{$15+$} & 0 & 261 & 69 & 434 & 74 & 1 & & \\
\hline & $1-400$ & 36 & 10 & 98 & 17 & 0.6 & $0^{f}$ & \\
\hline & $401-4000$ & 36 & 10 & 36 & 6 & 1.7 & 0.40 & \\
\hline & $4001-40000$ & 41 & 11 & 21 & 4 & 3.2 & 0.69 & \\
\hline & $40000+$ & 5 & 1 & 1 & 0 & 8.3 & 0.88 & \\
\hline & Total & 379 & & 590 & & & 0.40 & 0.12 \\
\hline \multirow[t]{6}{*}{ All } & 0 & 476 & 36 & 620 & 66 & $N A^{\pi}$ & & \\
\hline & $1-400$ & 124 & 9 & 144 & 15 & $N A^{\pi}$ & 0.29 & \\
\hline & $401-4000$ & 265 & 20 & 103 & 11 & $N A^{\pi}$ & 0.62 & \\
\hline & $4001-40000$ & 234 & 18 & 58 & 6 & $N A^{\pi}$ & 0.76 & \\
\hline & $40000+$ & 218 & 17 & 13 & 1 & $N A^{\pi}$ & 0.94 & \\
\hline & Total & 1317 & & 938 & & & 0.69 & 0.44 \\
\hline
\end{tabular}

${ }^{+}$Cases: with fever, axillary temperature $\geq 37.5^{\circ} \mathrm{C}$ at hospital visit.

${ }^{\ddagger}$ Controls: without fever, axillary temperature $<37.5^{\circ} \mathrm{C}$ at hospital visit and no reported history of fever during the preceding 3 days.

¥Percentage of patients in a certain parasite density class among cases/controls.

OR: odds-ratio (of fever at each stratum of parasite density and age, versus uninfected patients); AF: attributable fraction, calculated as:

$A F=\frac{O R-1}{O R}$

" ORs not calculated as overall AF calculated from results by age-category.

${ }^{E} A F$ by definition $\geq 0$.

PAF: population attributable fraction (calculated as showed in Table 3).

NA: Not applicable. 


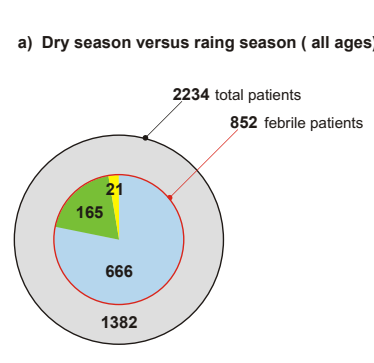

dry season

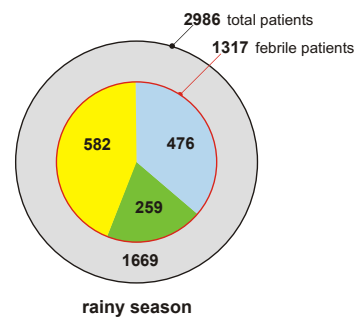

b) Rainy season: patients under 5 versus $\geq 5$ years old

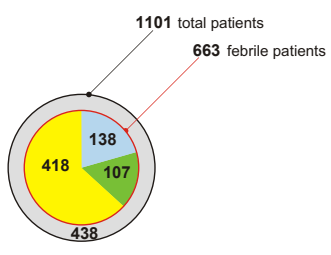

under 5

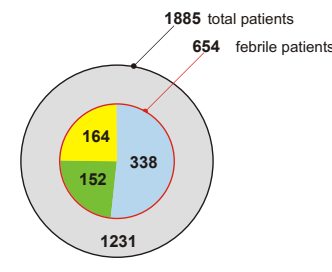

non febrile patients (primary data)

febrile not infected (primary data)

malaria attributable fevers (estimated)

febrile infected, not attributable (estimated)

(NB: areas are proportional to the respective populations)

Figure 3 Graphic representation of the patient population.

negative results at low parasite density might be relatively harmless. Niama-Meya et al in Uganda showed that the missed treatment for patients with a false negative malaria microscopy never resulted in severe disease [28].

Unfortunately, even at very high parasite density the RDT sensitivity was not $100 \%$. In one of our cases (a 6year-old child in the rainy season), who had malaria with very high parasite density $(>150,000 / \mu \mathrm{l})$, the RDT was negative and was confirmed as such by expert reading.
This worrisome occurrence, though rare, has been described by others for HRP-based tests [29], and might be explained by the pro-zone effect $[30,31]$. It makes clear-cut policies for patient management even more problematic. In our study RDTs showed a disappointing specificity, particularly in the rainy season. It is well known that HPR-based tests such as Paracheck may remain positive for weeks after disappearance of trophozoites. A recent study showed that $70 \%$ of these tests were still positive 35 days after appropriate treatment [22]. Moreover, the gold standard, the thick film, at the reading standard of our study, had a detection limit of about 50

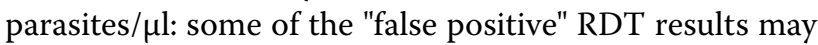
rather be false negative thick films. Studies with PCR have shown that RDT sensitivity for malaria infection may be higher than that of standard microscopy $[32,33]$.

\section{RDT accuracy for malaria - attributable fever}

In the dry season, after a positive RDT the probability for a fever to be attributable to malaria remains below $10 \%$. It is unquestionable that all patients with a positive RDT should be treated for malaria. A positive RDT, however, should not influence the treatment decision for other, potential causes of fever. In settings/seasons where malaria accounts for a negligible proportion of all fevers, RDTs are claimed to be most useful, and safe $[2,14]$. This study confirms that a negative test brings the probability of malaria down to virtually zero in the dry season. As only $28 \%$ of all RDTs were positive, a correct use of the test would avoid an unnecessary treatment in three quarters of the fevers. However, the risk involved in false positive results has not been given the attention it deserves. As it was previously reported, mortality was significantly higher in the dry season, when no death was due to malaria, and in one of the fatal cases the RDT had given a (false) positive result [4].

\section{Table 5: Diagnostic accuracy of RDT for malaria infection by parasite density (dry season)}

\begin{tabular}{|c|c|c|c|c|c|}
\hline \multirow{2}{*}{$\begin{array}{l}\text { Parasite density } \\
(/ \mu \mathrm{L})\end{array}$} & \multirow[b]{2}{*}{$\mathbf{N}$} & \multicolumn{2}{|l|}{ RDT Result } & \multirow[b]{2}{*}{ Sensitivity $(95 \% \mathrm{Cl})$} & \multirow[b]{2}{*}{ Specificity $(95 \% \mathrm{Cl})$} \\
\hline & & positive (n) & negative (n) & & \\
\hline 0 & 306 & 32 & 274 & & 90 \\
\hline $1-400$ & 50 & 38 & 12 & 76 & \\
\hline $401-4000$ & 28 & 27 & 1 & 96 & \\
\hline $4001-40000$ & 11 & 11 & 0 & 100 & \\
\hline $40000+$ & 5 & 5 & 0 & 100 & \\
\hline Not infected & 306 & 32 & 274 & & $90(86-92)$ \\
\hline Infected & 94 & 81 & 13 & $86(78-92)$ & \\
\hline Total & & $113(28 \%)$ & 287 (72\%) & & \\
\hline
\end{tabular}

PPV during dry season: 72\% (95\% Cl: 63 - 79\%), NPV during dry season: 95\% (95\% Cl: 92 - 97\%). 
Table 6: Diagnostic accuracy of RDT for malaria infection by parasite density and age group (rainy season)

\begin{tabular}{|c|c|c|c|c|c|c|}
\hline $\begin{array}{l}\text { Age } \\
\text { (years) }\end{array}$ & $\begin{array}{l}\text { Parasite density } \\
(/ \mu \mathrm{L})\end{array}$ & $\mathbf{N}$ & $\begin{array}{l}\text { RDT Result } \\
\text { positive (n) }\end{array}$ & negative (n) & Sensitivity $(95 \% \mathrm{CI})$ & Specificity $(95 \% \mathrm{Cl})$ \\
\hline \multirow[t]{6}{*}{$<1$} & 0 & 15 & 6 & 9 & & 60 \\
\hline & $1-400$ & 9 & 8 & 1 & 89 & \\
\hline & $401-4000$ & 18 & 17 & 1 & 94 & \\
\hline & $4001-40000$ & 22 & 22 & 0 & 100 & \\
\hline & $40000+$ & 20 & 20 & 0 & 100 & \\
\hline & Total & $84 \mathrm{fdf}(87 \%)(87 \%)$ & $73(87 \%)$ & $11(13 \%)$ & & \\
\hline \multirow[t]{6}{*}{$1-4$} & 0 & 57 & 22 & 35 & & 61 \\
\hline & $1-400$ & 18 & 15 & 3 & 83 & \\
\hline & $401-4000$ & 69 & 66 & 3 & 96 & \\
\hline & $4001-40000$ & 50 & 50 & 0 & 100 & \\
\hline & $40000+$ & 56 & 56 & 0 & 100 & \\
\hline & Total & 250 & 209 (84\%) & $41(16 \%)$ & & \\
\hline \multirow[t]{6}{*}{$5-14$} & 0 & 37 & 13 & 24 & & 65 \\
\hline & $1-400$ & 17 & 14 & 3 & 82 & \\
\hline & $401-4000$ & 30 & 28 & 2 & 93 & \\
\hline & $4001-40000$ & 20 & 20 & 0 & 100 & \\
\hline & $40000+$ & 24 & 23 & 1 & 96 & \\
\hline & Total & 128 & $98(77 \%)$ & 30 (23\%) & & \\
\hline \multirow[t]{6}{*}{$15+$} & 0 & 125 & 10 & 115 & & 92 \\
\hline & $1-400$ & 19 & 11 & 8 & 58 & \\
\hline & $401-4000$ & 20 & 18 & 2 & 90 & \\
\hline & $4001-40000$ & 23 & 23 & 0 & 100 & \\
\hline & $40000+$ & 1 & 1 & 0 & 100 & \\
\hline & Total & 188 & $63(34 \%)$ & $15(66 \%)$ & & \\
\hline \multirow[t]{6}{*}{ All } & 0 & 234 & 51 & 183 & & 78 \\
\hline & $1-400$ & 63 & 48 & 15 & 76 & \\
\hline & $401-4000$ & 137 & 129 & 8 & 94 & \\
\hline & $4001-40000$ & 115 & 115 & 0 & 100 & \\
\hline & $40000+$ & 101 & 100 & 1 & 99 & \\
\hline & Total & 650 & $443(68 \%)$ & $207(32 \%)$ & & \\
\hline \multirow[t]{2}{*}{ All } & Not infected & 234 & 51 & 183 & & $78(72-83)$ \\
\hline & Infected & 416 & 392 & 24 & $94(92-96)$ & \\
\hline
\end{tabular}

PPV during rainy season: $88 \%$ (95\% Cl: 85 - 91\%), NPV during rainy season: $88 \%$ (95\% Cl: 83 - 92\%).

The picture radically changes in the rainy season. Almost $90 \%$ of febrile children below 1 year, and almost $85 \%$ of those between 1 and 4 years, had a positive RDT (Table 6). After a positive test, a fever is very likely to be attributable to malaria (PPV $82 \%$ and $69 \%$, respectively) (Table 7), and even after a negative test the disease cannot be ruled out in either group, with a residual probability of
$16 \%$ and $10 \%$ (NPV $84 \%$ and $90 \%$ ), respectively. This raises concern especially for infants (6-11 months), because, even at very low parasite densities (the most likely to go undetected by the RDTs), the fever is almost invariably attributable to malaria. On the contrary, the negative test virtually excludes malaria in older children and adults. As, in contrast to children, two third of adults 
Table 7: Estimated diagnostic accuracy of RDT for malaria - attributable fever during the low and high transmission seasons

\begin{tabular}{|c|c|c|c|c|c|c|c|c|c|}
\hline \multirow{2}{*}{$\begin{array}{l}\text { Age } \\
\text { (years) }\end{array}$} & \multirow{2}{*}{$\begin{array}{l}\text { Febrile } \\
\mathrm{N}\end{array}$} & \multicolumn{2}{|c|}{ Malaria-attributable } & \multicolumn{2}{|c|}{ Not malaria-attributable } & \multirow{2}{*}{$\begin{array}{c}\text { SE } \\
\frac{a}{a+b}\end{array}$} & \multirow{2}{*}{$\begin{array}{l}\text { SP } \\
\frac{d}{c+d}\end{array}$} & \multirow{2}{*}{$\begin{array}{l}\text { PPV } \\
\frac{a}{a+c}\end{array}$} & \multirow{2}{*}{$\begin{array}{l}\mathbf{N P V} \\
\frac{d}{b+d}\end{array}$} \\
\hline & & TP (a) & $\mathbf{F N}(\mathbf{b})$ & FP (c) & TN (d) & & & & \\
\hline \multicolumn{10}{|c|}{ Low transmission (dry) season } \\
\hline$<1$ & 143 & 4.4 & 0.2 & 35.9 & 102.4 & 95 & 74 & 11 & 99.8 \\
\hline $1-4$ & 299 & 8.8 & 0.5 & 88.2 & 201.5 & 94 & 70 & 9 & 99.7 \\
\hline $5-14$ & 130 & 4.0 & 0.3 & 39.9 & 85.7 & 93 & 68 & 9 & 99.7 \\
\hline $15+$ & 280 & 2.5 & 0.3 & 46.2 & 230.9 & 89 & 83 & 5 & 99.9 \\
\hline All & 852 & 19.8 & 1.3 & 210.3 & 620.6 & 94 & 75 & 9 & 99.8 \\
\hline \multicolumn{10}{|c|}{ High transmission (rainy) season } \\
\hline$<1$ & 159 & 113.6 & 3.3 & 25.5 & 16.7 & 97 & 40 & 82 & 84 \\
\hline $1-4$ & 504 & 293.2 & 7.7 & 130.2 & 72.9 & 97 & 36 & 69 & 90 \\
\hline $5-14$ & 275 & 113.4 & 4.0 & 99.6 & 58.1 & 97 & 37 & 53 & 94 \\
\hline $15+$ & 379 & 45.7 & 1.4 & 74.4 & 257.4 & 97 & 78 & 38 & 99.4 \\
\hline All & 1317 & 565.8 & 16.4 & 329.7 & 405.1 & 97 & 55 & 63 & 96 \\
\hline
\end{tabular}

have a negative RDT result (Table 6), there appears to be a strong rationale for recommending a test-based policy for this age group, which would save a substantial proportion of unnecessary malaria treatments.

No published study as yet has attempted to assess RDT performances on malarial fever rather than on simple malaria infection. A recent study in Mali used a different approach to estimate RDT accuracy for clinical malaria: the latter was defined with empirical criteria, using as a case definition either the diagnosis by a clinician, or the presence of fever and of $\geq 2000$ plasmodia/ $\mu$ l at thick film [20]. Both case definitions are arguable, however. Malaria cannot be demonstrated on clinical grounds only [34,35]. The case definition based on parasite density is more adequate, but the optimal cut-off value should be assessed locally [36,37]. Any cut-off based method, however, leaves by definition a proportion of true malaria cases below the cut-off, and vice versa. The alternative method used in this study allows for an estimate of the actual number of malaria-attributable cases of fever within each stratum of parasite density.

\section{Strengths and weaknesses}

This study provides for the first time a formal estimate of how RDTs predict malaria-attributable fever in a popula- tion exposed to a highly variable malaria transmission intensity across seasons. Assessing the RDT accuracy only on infection is of course methodologically easier, but less informative. If RDTs are able to exclude malarial fever when negative, then they can be considered relatively safe even when missing some infections; if they are positive in a high proportion of febrile cases that are not attributable to malaria, then it is crucial to train health care providers not to use the positive test result as a pretext for exclusion of other possible causes of fever.

Some limitations must be duly acknowledged. First, the choice of a study setting based on health facilities may be questioned as not being representative of the general population. The use of RDTs in the country is not planned at community level but in health facilities only, and therefore the study population is a sample of the population which is actually targeted for the test-based policy.

Alternative methods to the AF approach to the case definition of malarial fever have been suggested [38]. However, almost all papers published since 1991 [26,3537,39-43] have used the AF calculation to this purpose. Of course, AF estimates can by no means be used as individual diagnostic criteria. 
Errors (confidence intervals for sensitivity, specificity and predictive values of RDT) are presented in our manuscript only for malaria infection and not for malariaattributable fever, due to a number of approximations for which the sampling error cannot be determined.

Finally, this study concerned but one out of the many commercially available RDTs that are currently under WHO scrutiny: several tests were more sensitive than Paracheck [22]. Would a more sensitive test change the main conclusions? The authors think it wouldn't. In the dry season, the test sensitivity on malaria-attributable fever was already optimal: a test with a better sensitivity, and equivalent specificity, for malaria infection would simply find more, clinically irrelevant, infections at low parasite density. In the rainy season, the same test might improve the safety of the test-based approach in young children, by identifying more cases of fever caused by malaria at the lower density strata, but at the expense of detecting also more cases of incidental parasitemia. As with Paracheck almost 90\% of test results were positive, with a more sensitive test this percentage would be close to $100 \%$, making it totally useless as a decisional tool.

\section{Policy implications}

RDTs appear to be most useful during the low transmission season: a negative test safely excludes malaria and would avoid most unnecessary treatments, if prescribers are convinced to rely on the negative result. However, they must also be aware of the low predictive value of a positive test: clinical guidelines should not limit to indicate malaria treatment in this case, but clearly recommend considering other life-threatening diseases, regardless of the test result.

In the high transmission season a negative test does not safely exclude malaria in children below 5 years and particularly so in infants. Moreover, most tests are positive in febrile children. Although cost implications are beyond the scope of this paper, it is clear that in such context the cost of the tests would be simply added to that of treatment, with a waste of resources. If these findings were confirmed by other studies, a RDT-based policy should not be recommended in similar contexts for young children. Therefore, in areas with great seasonal variation in malaria prevalence, the optimal policy might not be the same throughout the year. While for adults the indications would not differ, the same is not true for children. From an operational point of view, however, it is unrealistic to suggest a differentiated policy according to the season.

\section{Future research}

The cost-effectiveness of a RDT-based policy, in comparison with presumptive treatment, will be investigated, on the same study population, considering the test perfor- mances on malaria-attributable fever and not simply on infection.

\section{Conclusions}

Despite the study limitations that are duly acknowledged, these data provide enough evidence to suggest extreme caution before moving to a generalized test-based policy in all contexts. In areas similar to this study setting the test-based policy should probably remain restricted to older children and adults, at least until better and conclusive evidence on its safety in young children is produced. General guidelines on malaria diagnosis and treatment can be misleading, should they fail to take into account the local epidemiology.

\section{Additional material}

Additional file 1 Supplement Table 1. Calculation of diagnostic accuracy of RDT for malaria - attributable fever during the low transmission season

Additional file $\mathbf{2}$ Supplement Table $\mathbf{2}$. Calculation of diagnostic accuracy of RDT for malaria - attributable fever during the high transmission season

Additional file $\mathbf{3}$ Supplement Table 3. Diagnostic accuracy of RDT for malaria - attributable fever during the low transmission season based on individual level logistic regression models

Additional file 4 Supplement Table 4. Diagnostic accuracy of RDT for malaria - attributable fever during the high transmission season based on individual level logistic regression models

\section{Competing interests}

The authors declare that they have no competing interests.

\section{Authors' contributions}

ZB conceived the study design, wrote the study protocol, planned the training of research assistant and wrote the training manual (but for the laboratory). Trained research assistants in rainy season. Supervised enrolment and data collection in the field in rainy season. Concurred to data analysis. Wrote the draft and final version of the manuscript. SBS collaborated to the study design and writing of the study protocol. Contributed to draft versions, revised critically the manuscript.

JM performed statistical analysis (attributable fraction and RDT accuracy on malaria-attributable fever), contributed to draft versions, revised critically the manuscript and approved the final version. CP performed statistical analysis (logistic regression on factors affecting RDT accuracy for malaria infection), contributed to draft versions, revised critically the manuscript. AA trained research assistants in dry season. Supervised enrolment and data collection in the field in dry season. Performed bibliographic research. Revised critically the manuscript. FG trained research assistants in rainy season. Supervised enrolment and data collection in the field in rainy season. Performed bibliographic research. Revised critically the manuscript.

HT contributed to study design. Supervised enrolment and data collection in the field in both seasons. CL carried out first data analyses. Wrote a Master degree thesis (IMTA - Antwerp) reporting part of the paper findings for RDT accuracy for malaria infection. BN enrolled patients, trained and supervised research assistants, coordinated logistics in the field in both seasons. MG trained and supervised research assistants (laboratory) and local laboratory staff; wrote handbook for the research assistants (laboratory); performed microscopy; designed testing of reproducibility of microscopy reading. JVdE contributed with major inputs to the study design. Critically reviewed all draft versions, extensively collaborating for the final version.

All authors read and approved the final version of the manuscript.

\section{Acknowledgements}

The authors thank the patients who accepted to participate in the study, the personnel of the health centres involved and all the research assistants, as well as all the health staff of the "An Ka Heresso" Project (Progetto Mondo - MLAL) at 
the time of the field survey: Giuseppe Baracca (who first suggested a study on RDT), Klara Van den Ende, Annalisa Romeo, Mamadou Traore and Rosalie Midjour. We also thank Monica Degani and Barbara Paiola for their active collaboration to the training of the research assistants, Marleen Boelaert for support to data analysis and interpretation and Chris Whitty for critical reading of the manuscript. This study was funded by UNIDEA - UNICREDIT Foundation.

\section{Author Details}

${ }^{1}$ Centre for Tropical Diseases, S. Cuore Hospital, 37024 Negrar (Verona), Italy, 2 Projet AnKaHeresso, BP 292 Bobo Dioulasso, Burkina Faso, ${ }^{3}$ Centre National de Recherche et de Formation sur le Paludisme, Ministry of Health, B.P. 2208, Ouagadougou 01, Burkina Faso, ${ }^{4}$ Clinical Trials Unit, Prince Leopold Institute of Tropical Medicine, Nationalestraat 155, Antwerp, Belgium, 5 Institute of Genetic Medicine, European Academy (EURAC), Viale Druso 139100 Bozen/Bolzano, Italy, ${ }^{6}$ Centre Muraz, BP 390, Bobo-Dioulasso 01, Burkina Faso, ${ }^{7}$ Médecins Sans Frontières Italy, via Volturno 58, 00185 Rome, Italy and ${ }^{8}$ Department of Clinical Sciences, Prince Leopold Institute of Tropical Medicine, Nationalestraat 155, Antwerp, Belgium

Received: 1 May 2010 Accepted: 7 July 2010

Published: 7 July 2010

\section{References}

1. WHO: Guidelines for the treatment of malaria second edition. 2010 [http:// www.who.int/malaria/publications/atoz/9789241547925/en/index.html]. Geneva: WHO

2. D'Acremont V, Lengeler C, Mshinda H, Mtasiwa D, Tanner M, Genton B: Time to move from presumptive malaria treatment to laboratoryconfirmed diagnosis and treatment in African children with fever. PLOS Med 2009, 6:e252

3. English M, Reyburn H, Goodman C, Snow RW: Abandoning presumptive antimalarial treatment for febrile children aged less than five years--a case of running before we can walk? PLoS Med 2009, 6:e1000015.

4. Bisoffi Z, Sirima BS, Angheben A, Lodesani C, Gobbi F, Tinto H, Van den EJ: Rapid malaria diagnostic tests vs. clinical management of malaria in rural Burkina Faso: safety and effect on clinical decisions. A randomized trial. Trop Med Int Health 2009, 14:491-498.

5. Lubell Y, Reyburn H, Mbakilwa H, Mwangi R, Chonya S, Whitty CJ, Mills A: The impact of response to the results of diagnostic tests for malaria: cost-benefit analysis. BMJ 2008, 336:202-205.

6. Reyburn H, Mbakilwa H, Mwangi R, Mwerinde O, Olomi R, Drakeley C, Whitty CJ: Rapid diagnostic tests compared with malaria microscopy for guiding outpatient treatment of febrile illness in Tanzania: randomised trial. BMJ 2007, 334:403.

7. Belizario VY, Pasay CJ, Bersabe MJ, de Leon WU, Guerrero DM, Bugaoisan VM: Field evaluation of malaria rapid diagnostic tests for the diagnosis of P. falciparum and non-P. falciparum infections. Southeast Asian J Trop Med Public Health 2005, 36:552-561.

8. Gogtay NJ, Dalvi SS, Rajgor D, Chogle AR, Karnad DR, Ramdas M, Aigal U, Kshirsagar NA: Diagnostic and prognostic utility of rapid strip (OptiMal and Paracheck) versus conventional smear microscopy in adult patients of acute, uncomplicated P. falciparum malaria in Mumbai, India. J Assoc Physicians India 2003, 51:762-765.

9. Guthmann JP, Ruiz A, Priotto G, Kiguli J, Bonte L, Legros D: Validity reliability and ease of use in the field of five rapid tests for the diagnosis of Plasmodium falciparum malaria in Uganda. Trans R Soc Trop Med Hyg 2002, 96:254-257.

10. Hawkes M, Kain KC: Advances in malaria diagnosis. Expert Rev Anti Infect Ther 2007, 5:485-495.

11. Huong NM, Davis TM, Hewitt S, Huong NV, Uyen TT, Nhan DH, Cong ID: Comparison of three antigen detection methods for diagnosis and therapeutic monitoring of malaria: a field study from southern Vietnam. Trop Med Int Health 2002, 7:304-308.

12. Mboera LE, Fanello Cl, Malima RC, Talbert A, Fogliati P, Bobbio F, Molteni F: Comparison of the Paracheck-Pf test with microscopy, for the confirmation of Plasmodium falciparum malaria in Tanzania. Ann Trop Med Parasitol 2006, 100:115-122.

13. Mendiratta DK, Bhutada K, Narang R, Narang P: Evaluation of different methods for diagnosis of $P$. falciparum malaria. Indian J Med Microbiol 2006, 24:49-51.
14. Msellem MI, Martensson A, Rotllant G, Bhattarai A, Stromberg J, Kahigwa E, Garcia M, Petzold M, Olumese P, Ali A, Björkman A: Influence of rapid malaria diagnostic tests on treatment and health outcome in fever patients, Zanzibar: a crossover validation study. PLoS Med 2009, 6:e1000070.

15. Murray CK, Gasser RA JJ, Magill AJ, Miller RS: Update on rapid diagnostic testing for malaria. Clin Microbiol Rev 2008, 21:97-110.

16. Proux S, Hkirijareon L, Ngamngonkiri C, McConnell S, Nosten F: Paracheck-Pf: a new, inexpensive and reliable rapid test for $P$. falciparum malaria. Trop Med Int Health 2001, 6:99-101.

17. Rolland E, Checchi F, Pinoges L, Balkan S, Guthmann JP, Guerin PJ: Operational response to malaria epidemics: are rapid diagnostic tests cost-effective? Trop Med Int Health 2006, 11:398-408.

18. Singh N, Saxena A: Usefulness of a rapid on-site Plasmodium falciparum diagnosis (Paracheck PF) in forest migrants and among the indigenous population at the site of their occupational activities in central India. Am J Trop Med Hyg 2005, 72:26-29.

19. Singh N, Saxena A, Sharma VP: Usefulness of an inexpensive, Paracheck test in detecting asymptomatic infectious reservoir of Plasmodium falciparum during dry season in an inaccessible terrain in central India. J Infect 2002, 45:165-168.

20. Willcox ML, Sanogo F, Graz B, Forster M, Dakouo F, Sidibe O, Falquet J, Giani S, Diakite C, Diallo D: Rapid diagnostic tests for the home-based management of malaria, in a high-transmission area. Ann Trop Med Parasitol 2009, 103:3-16.

21. Bisoffi Z, Van den Ende J: Costs of treating malaria according to test results. BMJ 2008, 336:168-169.

22. Swarthout TD, Counihan H, Senga RK, van den Broek I: Paracheck-Pf accuracy and recently treated Plasmodium falciparum infections: is there a risk of over-diagnosis? Malar J 2007, 6:58.

23. Bruzzi P, Green SB, Byar DP, Brinton LA, Schairer C: Estimating the population attributable risk for multiple risk factors using case-control data. Am J Epidemiol 1985, 122:904-914.

24. Newcombe RG: Two-sided confidence intervals for the single proportion: comparison of seven methods. Stat Med 1998, 17:857-872.

25. Wang SJ, Lengeler C, Smith TA, Vounatsou P, Diadie DA, Pritroipa X, Convelbo N, Kientga M, Tanner M: Rapid urban malaria appraisal (RUMA) I: epidemiology of urban malaria in Ouagadougou. Malar J 2005, 4:43.

26. McGuinness D, Koram K, Bennett S, Wagner G, Nkrumah F, Riley E: Clinical case definitions for malaria: clinical malaria associated with very low parasite densities in African infants. Trans R Soc Trop Med Hyg 1998, 92:527-531.

27. Anonymous: Malaria Rapid Diagnostic Test Performance. Results of WHO product testing of malaria RDTs: Round 1 (2009). TDR 2009:100, (ISBN: 9789241598071 - DOI: 10.2471/TDR.09.978-924-1598071)

28. Njama-Meya D, Clark TD, Nzarubara B, Staedke S, Kamya MR, Dorsey G: Treatment of malaria restricted to laboratory-confirmed cases: a prospective cohort study in Ugandan children. Malar J 2007, 6:7.

29. Drakeley C, Reyburn H: Out with the old, in with the new: the utility of rapid diagnostic tests for malaria diagnosis in Africa. Trans $R$ Soc Trop Med Hyg 2009, 103:333-337.

30. Risch L, Bader M, Huber AR: False negative quick malaria test. Schweiz Med Wochenschr 1999, 129:1002.

31. Gillet P, Mori M, Van EM, Van den Ende J, Jacobs J: Assessment of the prozone effect in malaria rapid diagnostic tests. Malar J 2009, 8:271.

32. Nicastri E, Bevilacqua N, Sane Schepisi M, Paglia MG, Meschi S, Ame SM, Mohamed JA, Mangi S, Fumakule R, Di Caro A, Capobianchi MR, Kitua A, Molteni F, Racalbuto V, Ippolito G: Accuracy of malaria diagnosis by microscopy, rapid diagnostic test, and PCR methods and evidence of antimalarial overprescription in non-severe febrile patients in two Tanzanian hospitals. Am J Trop Med Hyg 2009, 80:712-717.

33. Stauffer WM, Cartwright CP, Olson DA, Juni BA, Taylor CM, Bowers $\mathrm{SH}$, Hanson KL, Rosenblatt JE, Boulware DR: Diagnostic performance of rapid diagnostic tests versus blood smears for malaria in US clinical practice. Clin Infect Dis 2009, 49:908-913.

34. Chandramohan D, Jaffar S, Greenwood B: Use of clinical algorithms for diagnosing malaria. Trop Med Int Health 2002, 7:45-52.

35. Mwangi TW, Mohammed M, Dayo H, Snow RW, Marsh K: Clinical algorithms for malaria diagnosis lack utility among people of different age groups. Trop Med Int Health 2005, 10:530-536.

36. Dicko A, Mantel C, Kouriba B, Sagara I, Thera MA, Doumbia S, Diallo M, Poudiougou B, Diakite M, Doumbo OK: Season, fever prevalence and 
pyrogenic threshold for malaria disease definition in an endemic area of Mali. Trop Med Int Health 2005, 10:550-556.

37. Schellenberg JR, Smith T, Alonso PL, Hayes RJ: What is clinical malaria? Finding case definitions for field research in highly endemic areas. Parasitol Today 1994, 10:439-442.

38. Ochola LB, Vounatsou P, Smith T, Mabaso ML, Newton CR: The reliability of diagnostic techniques in the diagnosis and management of malaria in the absence of a gold standard. Lancet Infect Dis 2006, 6:582-588.

39. Bejon P, Mwangi T, Lowe B, Peshu N, Hill AV, Marsh K: Clearing asymptomatic parasitaemia increases the specificity of the definition of mild febrile malaria. Vaccine 2007, 25:8198-8202.

40. Rogers WO, Atuguba F, Oduro AR, Hodgson A, Koram KA: Clinical case definitions and malaria vaccine efficacy. J Infect Dis 2006, 193:467-473.

41. Rougemont A, Breslow N, Brenner E, Moret AL, Dumbo O, Dolo A, Soula G, Perrin L: Epidemiological basis for clinical diagnosis of childhood malaria in endemic zone in West Africa. Lancet 1991, 338:1292-1295.

42. Saute F, Aponte J, Almeda J, Ascaso C, Abellana R, Vaz N, Dgedge M, Alonso P: Malaria in southern Mozambique: malariometric indicators and malaria case definition in Manhica district. Trans R Soc Trop Med Hyg 2003, 97:661-666.

43. Smith T, Schellenberg JA, Hayes R: Attributable fraction estimates and case definitions for malaria in endemic areas. Stat Med 1994, 13:2345-2358

doi: $10.1186 / 1475-2875-9-192$

Cite this article as: Bisoffi et al., Accuracy of a rapid diagnostic test on the diagnosis of malaria infection and of malaria - attributable fever during low and high transmission season in Burkina Faso Malaria Journal 2010, 9:192

Submit your next manuscript to BioMed Central and take full advantage of:

- Convenient online submission

- Thorough peer review

- No space constraints or color figure charges

- Immediate publication on acceptance

- Inclusion in PubMed, CAS, Scopus and Google Scholar

- Research which is freely available for redistribution

Submit your manuscript at www.biomedcentral.com/submit
Ciomed Central 